\title{
DESARROLLO FONOLÓGICO-FONÉTICO EN UN GRUPO DE NIÑOS ENTRE 3 Y 5, 11 AÑOS
}

\section{Phonetic-phonological child development in a group of children between 3 and 5.11-year old}

\author{
Pilar Vivar ${ }^{(1)}$, Hernán León ${ }^{(2)}$
}

\begin{abstract}
RESUMO
Objetivo: conocer cómo los niños adquieren el sistema fonológico-fonético de su lengua materna y describir la cronología de secuencia de adquisición del repertorio fonético-fonológico en la muestra. Métodos: la muestra está compuesta por 72 sujetos dividida en seis grupos etáreos de seis meses cada uno. Se le aplicó el "Cuestionario para la Evaluación de la Fonología Infantil" (CEFI) que evaluó la articulación de las consonantes en sus todas sus posiciones mediante una tarea de nombrado. Los resultados de cada grupo etáreo fueron comparados en cada segmento para observar la secuencia de adquisición de los sonidos según la edad de los informantes. Resultados: se observó que el rendimiento general de las consonantes evidenció un aumento en el porcentaje de emisión normal en relación al avance en la edad de los sujetos. Los fonemas $/ f /-/ \mathrm{s} /-/ \mathrm{x} /$ y el grupo de líquidas /l/- /lh/ - /r/ fueron los segmentos que presentaron mayor dificultad articulatoria. Las nasales $/ \mathrm{m} /-/ \mathrm{n} /-/ \mathrm{nh} /$ y las sordas no continuas $/ \mathrm{p} /-/ \mathrm{t} /-/ \mathrm{tch} /-/ \mathrm{k} /$ fueron los segmentos con mayor porcentaje de articulación normal. Conclusiones: a los 3 años los sujetos tienen sobre un $80 \%$ de articulación normal en las consonantes.Las mayores dificultades de adquisición se manifestaron en las consonantes fricativas $/ \mathrm{s} / \mathrm{y} / \mathrm{x} / \mathrm{y}$, sobre todo, en la consonante $/ \mathrm{r} /$. A la inversa, las consonantes que menos dificultades de adquisición presentaron fueron las nasales y las oclusivas.
\end{abstract}

DESCRIPTORES: Lenguaje Infantil; Desarrollo del Lenguaje; Articulación Infantil; Fonética

\section{INTRODUCCIÓN}

La adquisición fonológica es un proceso complejo, por lo que una teoría por sí sola no puede cubrir de manera satisfactoria todos los factores que intervienen en él. Una teoría de adquisición fonológica, debería dar cuenta de cómo los niños adquieren y llegan a dar uso correcto a los patrones sonoros de su lengua materna. Tradicionalmente, en teoría fonológica se reconocen dos grandes enfoques: uno es la fonémica taxonómica y el otro la fonología generativa. Por un lado, la fonémica taxonómica se encuentra relacionada con los preceptos estructuralistas de la Escuela de Praga, en donde se concibe al fonema como una unidad

\footnotetext{
(1) Profesora de español; Magíster en Linguística; Universidad de Concepción, Chile.

(2) Fonoaudiólogo; Magíster en Linguística; Universidad de Concepción, Chile.

Conflito de interesse: INEXISTENTE
}

mínima capaz de producir contrastes. Desde este enfoque los niños adquirirían el sistema fonológico mediante la oposición de rasgos pertinentes. Por otro lado, la fonología generativa profundiza lo planteado anteriormente al proponer que cada sonido posee varios elementos constituyentes o rasgos, por ejemplo/d/: [+oclusiva], [+postdental], [+sonora]. Este enfoque sienta las bases de lo que posteriormente sería la "fonología natural" la cual describe el desarrollo fonológico infantil en función del lenguaje adulto por medio de los Procesos Fonológicos de Simplificación.

Otras teorías desarrolladas han sido: a) Modelos cognitivos; b) Teorías de la inmadurez fonética; c) Hipótesis de la autoorganización; d) Modelo de principios y parámetros ${ }^{1}$. Durante los años 70 y 80 surgen los modelos de tipo cognitivo, los cuales atribuyen un papel activo y creador al niño. Desde esta perspectiva los procesos fonológicos son inventados gracias a una serie de estrategias que permiten al pequeño crear las reglas de construcción. En 
otras palabras, los niños son protagonistas activos de su desarrollo fonológicco, pues ellos mismos revisan, y testean su fonología en función de sus expresiones ${ }^{2}$.

Una teoría relacionada con la percepción es la de inmadurez fonética, la cual sugiere que a pesar de que los bebés pueden percibir y diferenciar finos rasgos fonéticos, no pueden percibirlos todos; es más, el hecho de que un niño pueda recordar sutiles diferencias fonético-acústicas no significa que tenga la capacidad para almacenarlas y recodarlas después ${ }^{1}$.

Durante la década de los 80 se configura una línea teórica con bases físicas y biológicas. Entre los aspectos más destacables es que identifica en el período prelingüístico una serie de patrones regulares y universales considerando que las diferencias individuales tendrían una base biológica. Una característica relevante en la pronunciación infantil sería su menor velocidad en relación a la pronunciación de los adultos y agrega que esto sería atribuible a que un niño menor de tres años no posee la velocidad de conducción nerviosa adecuada ni el peso del cerebro de un adolescente ${ }^{3}$.

Entre las teorías más contemporáneas se puede señalar la autosegmental o métrica, desde esta perspectiva el análisis del desarrollo fonológico se realiza considerando niveles de representación autónomos y organizados. En este análisis se incorporan aspectos prosódicos y además los rasgos fonéticos son analizados más allá de unidades segmentales como la sílaba y la palabra 4 .

En el marco lingüístico más reciente, se encuentra la teoría de la optimalidad la cual sugiere que los sucesivos estados de la gramática infantil y la gramática adulta comparten un conjunto universal de restricciones, pero difieren en la jerarquía de las mismas, y que el proceso de adquisición fonológica consiste en una rejerarquización de las restricciones ${ }^{5}$.

La mayoría de los estudios sobre adquisición fonológica han establecido rangos etáreos y sobre ellos se han establecido los segmentos que estarían "adquiridos" para tales rangos. Lo anterior ha permitido establecer mediante frecuencias de aparición, el orden de dificultad en la producción de los segmentos. La importancia de este tipo de investigaciones es que permiten establecer el patrón de adquisición normal y de esta manera poder identificar a niños con retrasos o trastornos en el desarrollo de su sistema fonológico ${ }^{6,7}$. Entre las pruebas existentes para medir el rendimiento fonético-fonológico infantil en Chile, se encuentra el Test de Evaluación de los Procesos Fonológicos de Simplificación (TEPROSIF) ${ }^{8}$ el cual, enmarcado en la fonología natural, mide la aparición de proce- sos fonológicos de simplificación en la producción articulatoria. Durante el año 2004, se llevó a cabo un trabajo de investigación en el que se comprobó que el "Cuestionario para la evaluación de la Fonología Infantil (CEFI)" era aplicable a hablantes del español de Chile. En dicha investigación el CEFI, aplicado a una muestra local, dio cuenta de las diferencias en el desarrollo fonológico entre niños de distintos niveles socioculturales ${ }^{9}$. La investigación anterior también permitió efectuar adaptaciones dialectales que permiten la utilización de este instrumento para evaluar el desempeño fonológico infantil en Chile.

En la siguiente investigación se establece como objetivo general conocer el desarrollo fonético-fonológico en un grupo de niños hablantes del dialecto español de Chile y como objetivo específico describir la cronología de secuencia de adquisición del repertorio fonético-fonológico.

\section{MÉTODOS}

La muestra está compuesta por 72 sujetos dividida en seis grupos etáreos de seis meses, evaluándose en cada uno a 6 hombres y 6 mujeres haciendo un total de 12 sujetos por grupo etáreo. La distribución de la edad se observa en la Tabla 1.

Los sujetos de esta investigación pertenecen al nivel socioeconómico medio-alto. Para procurar lo anterior, se decide trabajar sólo con jardines infantiles particulares y subvencionados por el estado. La selección de la muestra se realizó de acuerdo a: normalidad de la articulación (previo examen fonoaudiológico); - edad y género de los informantes.

El "Cuestionario para la Evaluación Infantil" CEFI consta de 49 láminas, en su mayoría sustantivos. En cada lámina se evalúa a más de una consonante (Figura 1). Como criterio general se seleccionaron palabras e imágenes pertenecientes al vocabulario infantil y a la realidad del dialecto español de Chile (Figura 2). Las respuestas de los sujetos fueron transcritas fonológicamente de manera simultánea a la aplicación del test. Como apoyo para transcripción, las entrevistas son grabadas para una posterior revisión fonética.

Tabla 1 - Distribución etarea de los sujetos

\begin{tabular}{c|c}
\hline Grupo etáreo & Edad \\
\hline Grupo I & $3,0-3,5$ \\
Grupo II & $3,6-3,11$ \\
Grupo III & $4,0-4,5$ \\
Grupo IV & $4,6-4,11$ \\
Grupo V & $5,0-5,5$ \\
Grupo VI & $5,6-5,11$ \\
\hline
\end{tabular}




\begin{tabular}{|c|c|c|c|}
\hline Posición & Oclusivas & Fricativas / Africadas & Sonantes \\
\hline Inicial & $\begin{array}{l}/ \mathrm{p} /-/ \mathrm{t} / /-/ \mathrm{k} / \\
/ \mathrm{b} /-/ \mathrm{d} / /-/ \mathrm{g} /\end{array}$ & $\begin{array}{c}|\mathrm{ff} /-| \mathrm{s} /-|\mathrm{x} /-| \mathrm{j} / \\
/ \mathrm{t} J /\end{array}$ & $\begin{array}{c}/ \mathrm{m} /-/ \mathrm{n} /-/ \mathrm{n} / \\
/ \mathrm{r} /-/ \mathrm{II} /\end{array}$ \\
\hline Media & $\begin{array}{l}I \mathrm{p} /-/ \mathrm{t} / /-/ \mathrm{k} / \\
/ \mathrm{b} /-/ \mathrm{d} / / / \mathrm{g} /\end{array}$ & $\begin{array}{c}|\mathbf{f} /-| \mathbf{s} /-|\mathbf{x}|-|\mathrm{j}| \\
|\mathbf{t} J|\end{array}$ & $\begin{array}{c}|\mathrm{m} /-| \mathrm{n} /-\mid \mathrm{n} / \\
|\mathrm{r} /-| \mathrm{r} /-/ I /\end{array}$ \\
\hline Final & $/ \mathrm{d} /$ & $|\mathbf{s}|-|\mathbf{x}|$ & $/ \mathrm{n} /-/ \mathrm{I} /-/ \mathrm{r} /$ \\
\hline
\end{tabular}

Figura 1 - Lista de fonemas consonánticos evaluados en CEFI

\begin{tabular}{|l|l|l|l|l|l|l|}
\hline 1. Perro & 8. Niño & 15. Caja & 22. Leche & 29. Baila & 36. Cárcel & 43. Clavos \\
\hline 2. Ojo & 9. Peineta & 16. Llave & 23. Limón & 30. Torta & 37. Chico & 44. Flecha \\
\hline 3. Amarillo & 10. Jirafa & 17. Mosca & 24. Falda & 31. Dientes & 38. Resbaló & 45. Enfermo \\
\hline 4. Sopa & 11. Rueda & 18. Naranja & 25. Piano & 32. Tapa & 39. Brazo & 46. Inyección \\
\hline 5. Reloj & 12. Estrella & 19. Gorro & 26. Jaula & 33. Trompeta & 40. Globo & 47. Hombre \\
\hline 6.Gato & 13. Bolsa & 20. Tambor & 27. Blusa & 34. Fuego & 41. Pluma & 48. Ombligo \\
\hline 7. Muñeca & 14. Árbol & 21. Doctor & 28. Chofer & 35. Ciudad & 42. Cuadro & 49. Alto \\
\hline
\end{tabular}

Figura 2 - Láminas evaluadas por CEFI

Para contabilizar y evaluar las emisiones de los sujetos la prueba CEFI organiza a los fonemas en: consonantes aisladas, grupos consonánticos y diptongos. Las consonantes, a su vez, son organizadas en cinco grupos según el modo articulatorio:

- /b/, /d/, /j/, /g/ : sonoras orales

- /p/, /t/, /tt/, / /k/: sordas no continuas

- /f///s/, /x/: sordas continuas

- /m/, /n/, /n/: nasales

- /r/, /r/, /l/: laterales

La prueba evalúa cada articulación mediante cuatro claves $\mathbf{N}$ (normal), $\mathbf{M}$ (modificado), $\mathbf{S}$ (sustituido), $\mathbf{O}$ (omisión) y ? (duda por parte del investigador). La modificación corresponde a un cambio de tipo fonético dentro del rango articulatorio del fonema. Por otro lado, la sustitución se produce cuando un fonema es sustituido por otro. El criterio principal que se utiliza para considerar que existe un cambio de tipo fonológico está relacionado con el carácter distintivo del fonema. La omisión se produce cuando el informante no emite el fonema esperado.

El cálculo del rendimiento articulatorio en cada grupo etáreo se realizó en función del número de veces que cada fonema aparece en el test. Por ejemplo, si /b/ es evaluado 5 veces en CEFI entonces el total de producciones esperado para ese fonema será el número de sujetos por grupo etáreo, 12 , multiplicado por 5 , el resultado será 60 que indica el $100 \%$ de emisiones para dicho fonema. Siguiendo la estructura del test, se contabiliza luego el total para cada grupo de fonemas y a partir de ese valor se estima el \% de producciones normales, modificadas, sustituidas y omitidas (Figura 3 ).

\section{RESULTADOS}

El rendimiento general de las consonantes evidenció un aumento en el porcentaje de emisión normal en relación al avance en la edad de los informantes. Esta correlación también se manifestó en la disminución paulatina de los fenómenos articulatorios que el test CEFI considera como no normales.

La sustitución correspondió al fenómeno no normal con mayor porcentaje de aparición en la muestra y su presencia se mantuvo aún en los informantes con mayor edad ( $\mathrm{V}$ y VI grupos etáreos). La modificación tuvo una aparición homogénea en cuanto al porcentaje alcanzado en cada grupo etáreo. En la Figura 4 se puede apreciar el comportamiento general de las consonantes aisladas en cada grupo de sujetos.

De manera particular, el grupo de nasales presentó un $100 \%$ de articulación normal a partir del II grupo etáreo. Este alto rendimiento convierte a las nasales junto a las sordas no continuas en las consonantes con mayor porcentaje de emisión normal (Figura 5).

Los fonemas $/ \mathrm{p} /-/ \mathrm{t} / \mathrm{-} / \mathrm{t} / \mathrm{-} / \mathrm{k} / \mathrm{o}$ sordas no continuas presentaron sobre un $90 \%$ de articulación normal en todos los grupos etáreos. La sustitución se evidenció como el fenómeno articulatorio con mayor porcentaje luego de la emisión normal, no obstante su presencia fue disminuyendo a medida que aumentó la edad de los sujetos. Las oclusivas 


\begin{tabular}{|c|c|c|c|}
\hline \multicolumn{2}{|l|}{ Segmento } & $\mathrm{N}^{\circ}$ de aparición (100\%) & Total \\
\hline \multirow{4}{*}{ Sonoras orales } & $/ \mathrm{b} /$ & 60 & \multirow{4}{*}{204} \\
\hline & $/ \mathrm{d} /$ & 60 & \\
\hline & $\mid \mathrm{j} /$ & 36 & \\
\hline & $\mathrm{lg} /$ & 48 & \\
\hline \multirow{4}{*}{$\begin{array}{l}\text { Sordas no } \\
\text { continuas }\end{array}$} & $/ \mathrm{p} /$ & 60 & \multirow{4}{*}{240} \\
\hline & $/ \mathrm{t} /$ & 72 & \\
\hline & $/ \mathrm{t}] /$ & 48 & \\
\hline & $/ \mathrm{k} /$ & 60 & \\
\hline \multirow{3}{*}{ Sordas continuas } & $/ \mathrm{f} /$ & 48 & \multirow{3}{*}{180} \\
\hline & $/ \mathrm{s} /$ & 72 & \\
\hline & $|x|$ & 60 & \\
\hline \multirow{3}{*}{ Nasales } & $/ \mathrm{m} /$ & 60 & \multirow{3}{*}{156} \\
\hline & $/ \mathrm{n} /$ & 72 & \\
\hline & $/ \mathrm{n} /$ & 24 & \\
\hline \multirow{3}{*}{ Líquidas } & $|r|$ & 36 & \multirow{3}{*}{228} \\
\hline & $\mid r /$ & 96 & \\
\hline & /I/ & 96 & \\
\hline
\end{tabular}

Figura 3 - Emisión esperada por segmento

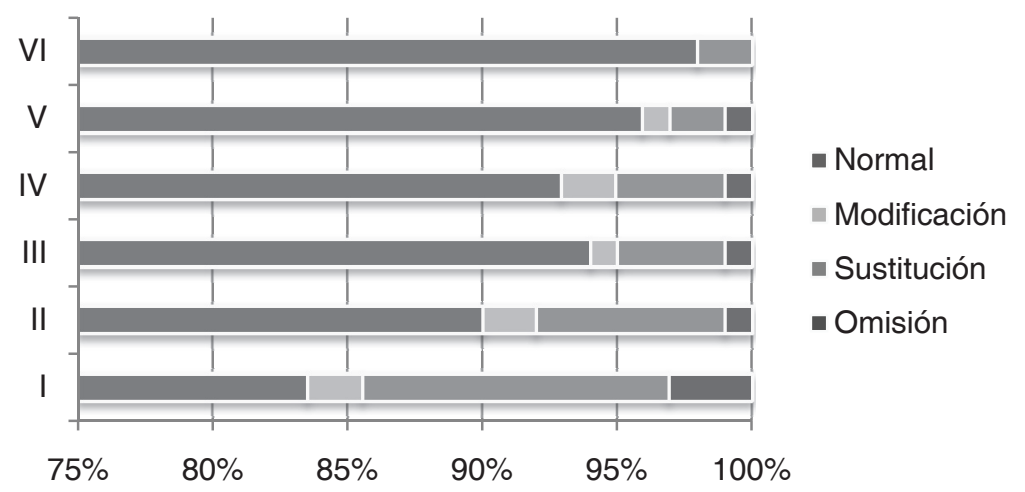

Figura 4 - Consonantes aisladas

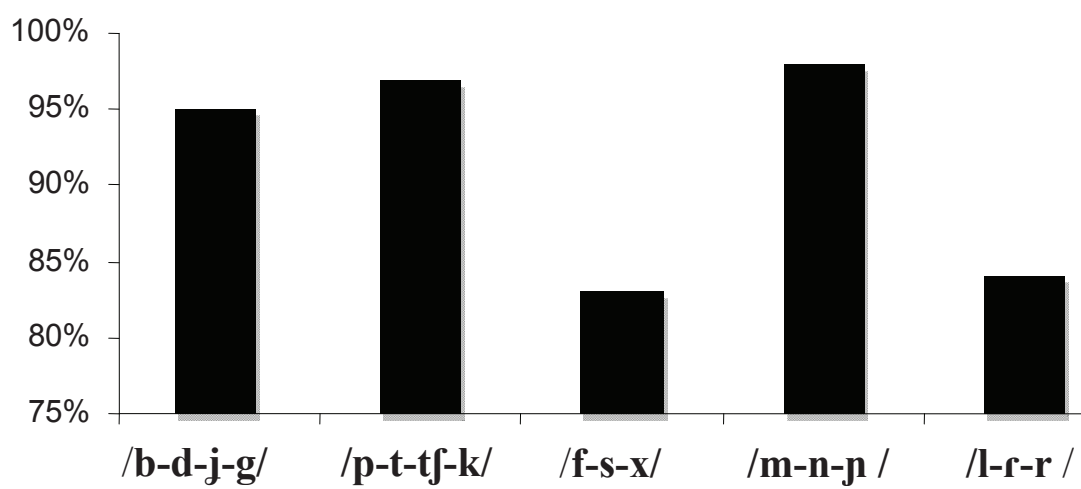

Figura 5 - Porcentaje de emisión normal por grupo de consonantes 
sordas /p, t, k/ fueron las que presentaron mayor porcentaje de emisión normal. Por otro lado, el único fonema de este grupo que manifestó casos de sustitución fue la africada sorda /tt/ por los siguientes fonemas: /t, g, s, r/: “chofer" [to.'per] [go.'fér], [so.'fér], [ro.'fer]); "flecha" ['pe.ta], ['fe.sa]; y "chico" ['ti.ko], ['si.ko].

Por su parte, los fonemas $/ \mathrm{b} /-/ \mathrm{d} /-/ \mathrm{j} /-/ \mathrm{g} / \mathrm{o}$ sonoras orales presentaron sobre un $80 \%$ de emisión normal en los 6 grupos etáreos. Tanto la bilabial sonora /b/ como la fricativa sonora/j/ fueron las que presentaron mayor porcentaje de articulación normal. Por el contrario, los fonemas $/ \mathrm{d} / \mathrm{y} / \mathrm{g} /$ fueron aquellos que presentaron más casos de sustitución y omisión, particularmente la oclusiva sonora /d/.

En las sordas no continuas constituidas por los fonemas $/ f /-/ s /-/ x /$, se evidenció un aumento en el porcentaje de emisión normal a medida que avanzó la edad de los informantes, no obstante no se logró el $100 \%$ de emisión normal en el último grupo etáreo (Figura 6). En este grupo de consonantes se manifestaron los tres fenómenos articulatorios no normales (modificación, sustitución y omisión) y la presencia de ellos se mantuvo hasta en los sujetos con mayor edad de la muestra. Los casos de modificación se presentaron sólo en el fonema /s/ en donde mayoritariamente es articulado por su alófono interdental $[\theta]$. Por su parte, la sustitución se presentó en las tres fricativas. En la primera de ellas, /f/, la sustitución se produjo principalmente por el fonema / $/$ / en la lámina "fuego", dando como resultado [xwé.yo].También se evidenciaron sustituciones de /f/ por /p/ y /s/ en las láminas "jirafa": [xi.'la.pa], [ni.'fa.sa]; y en "chofer" por [to.'per]. Cabe señalar, que este tipo de articulaciones se presentó sólo en los sujetos de menor edad.

En el fonema /s/, la sustitución aparece con mayor porcentaje en el grupo etáreo I, no obstante dicho fenómeno desaparece por completo en 4 de los 5 grupos posteriores. La sustitución de /s/ se evidenció por los fonemas /tt/, /t/, /p/ y /r/ en: "blusa" ['blu.tfa], [ru.pa]; "dientes": ['djen.tetf]; "sopa": ['to.pa]; "brazo": ['ba.to]; "ciudad": [t]u.'dah], entre otros ejemplos. Por su parte, la sustitución en el fonema $/ x /$ no presentó una disminución en relación al avance de los grupos etáreos. El fenómeno anterior se producjo mayoritariamente en la lámina "reloj", en donde los sujetos sustituyeron la fricativa velar por fonemas oclusivos como /k/ y /t/: [re.'lok], [re.'lot]. Esta oclusivización de la fricativa también se evidenció, aunque con menos frecuencia, en posición inicial en [táu.la] por "jaula" y en posición V_V en ['ta.ta] y ['o.to] por "caja" y "ojo", respectivamente.

La omisión se presentó en los fonemas /s/ y / $\mathrm{x} /$. En el primero de ellos, este fenómeno se produjo en posición final en láminas como "dientes" y "clavos", su porcentaje de aparición es mayor en el primer grupo etáreo, dicho fenómeno disminuye en los grupos etáreos posteriores para desaparecer por completo en el último grupo de sujetos. En el último fonema de este grupo de consonantes fricativas, es decir, $\mid x /$, los casos de omisión se presentaron en posición final en la lámina "reloj", se observa que este disminuye de acuerdo avanza los grupos etáreos.

Finalmente, en el grupo de líquidas $(/ / /-/ r /-/ r /)$ las articulaciones no normales se concentraron mayoritariamente en las vibrantes. El fenómeno articulatorio no normal con mayor porcentaje de aparición fue la sustitución sobre todo en $/ \mathrm{r} /$; mientras que, el menor, fue la omisión. La modificación ocupó el segundo lugar en cuanto a frecuencia y, nuevamente, los mayores porcentajes se encuentran en la vibrante múltiple.

La modificación del fonema / $/$ / se presentó por la articulación del sonido [u]. La sustitución, por su parte, presentó un comportamiento regular en los grupos etáreos, es decir, su aparición disminuye a medida que aumenta la edad de los informantes. Los casos de sustitución en /r/ se produjeron por la articulación de los fonemas /j/, /l/, /d/, /f/ y /n/.

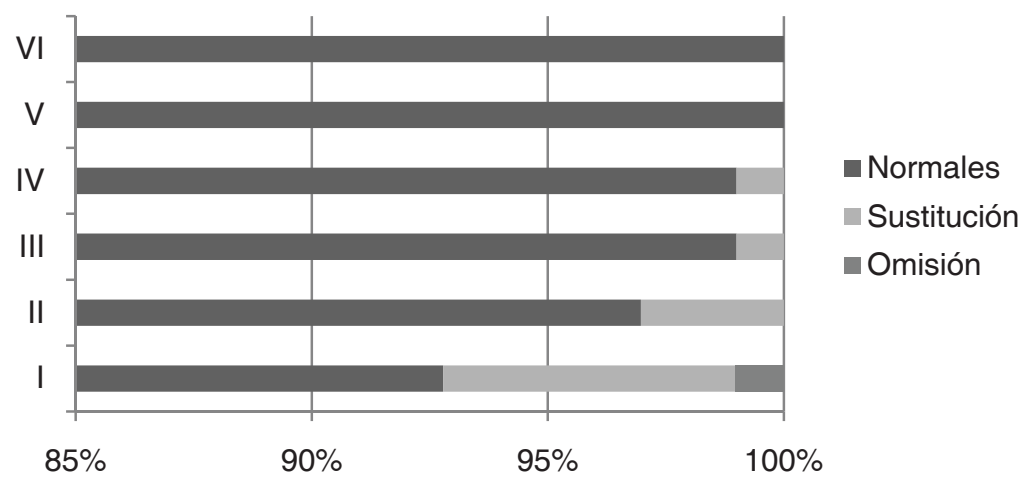

Figura 6 - Sordas no Continuas

Rev. CEFAC. 2009 Abr-Jun; 11(2):190-198 
Particularmente, / / y /// fueron las sustituciones más frecuentes. Por su parte, en el fonema/r/ (láminas: "perro", "gorro", "resbaló", "rueda", "reloj", "chofer", "tambor" y "doctor") los casos de modificación se presentaron por la articulación de los sonidos [.] y [ı] dicha articulación presentó un comportamiento irregular según los grupos etáreos, alcanzando su mayor porcentaje en los grupos III y IV (con un $10 \%$ ). Las sustituciones observadas al articular /r/ fueron: /d/, /r/, /j/, /l/, /s/, /h/, /k/, /t/, /b/ y /g/, y específicamente $/ \mathrm{d} / /, / \mathrm{r} / \mathrm{/j} /$ son las que presentaron mayor frecuencia. Esta articulación posee su mayor porcentaje en el primer grupo de informantes, en el segundo grupo etáreo la sustitución disminuyó a casi la mitad. Por su parte, la omisión en /r/ posee su mayor porcentaje en el primer grupo de sujetos.
En el fonema /// se presentó el menor porcentaje de emisiones modificadas y sustituidas en comparación a otros fonemas de este grupo. En los tres últimos grupos etáreos estas emisiones desaparecieron por completo. Entre las consonantes líquidas, la vibrante múltiple es aquella que presentó mayor dificultad para los informantes, lo anteriormente expuesto puede observarse en la Figura 7.

Para finalizar la presentación de los resultados, se ha confeccionado la Tabla 2, en donde se presenta de manera descriptiva el porcentaje de aparición de los fonemas en cada grupo etáreo. Esto permitirá al lector observar el avance en el dominio de cada fonema a medida que aumenta la edad de los sujetos.

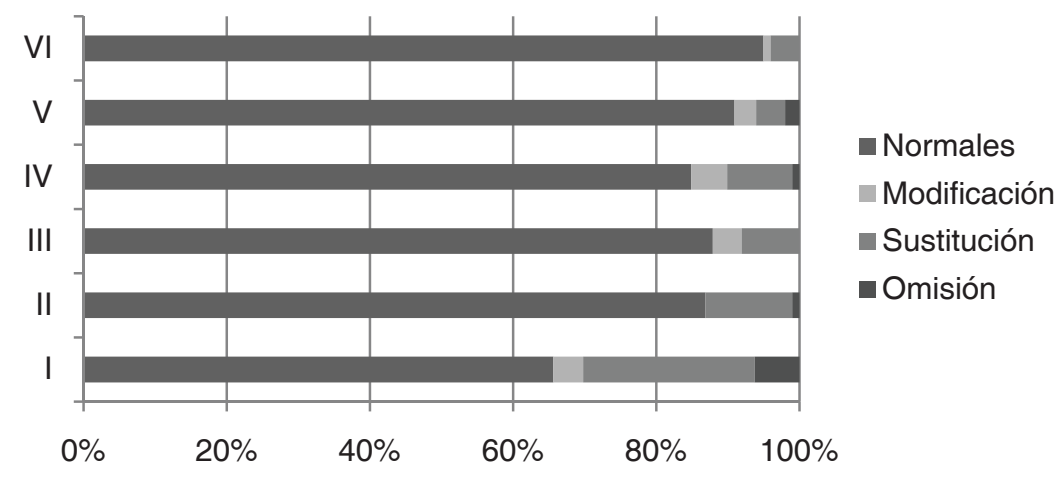

Figura 7 - Líquidas

Tabla 2 - Secuencia de adquisición de los fonemas

\begin{tabular}{|c|c|c|c|c|c|c|}
\hline 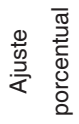 & $\begin{array}{c}\text { I Grupo } \\
3,0-3,5 \text { años }\end{array}$ & $\begin{array}{c}\text { II Grupo } \\
\text { 3, } 6-3,11 \text { años }\end{array}$ & $\begin{array}{c}\text { III Grupo } \\
4,0-4,5 \text { años }\end{array}$ & $\begin{array}{c}\text { IV Grupo } \\
4,5-4,11 \text { años }\end{array}$ & $\begin{array}{c}\text { V Grupo } \\
5,0-5,5 \text { años }\end{array}$ & $\begin{array}{c}\text { VI Grupo } \\
5,5-5,11 \text { años }\end{array}$ \\
\hline $100 \%$ & $/ \mathrm{p} / / \mathrm{t} / / \mathrm{m} /$ & $/ \mathrm{b} / / \mathrm{p} / / \mathrm{t} / / \mathrm{m} / / \mathrm{n} / / \mathrm{n} /$ & $\begin{array}{l}/ \mathrm{b} / / \mathrm{j} / / \mathrm{p} / \mathrm{t} / / \mathrm{k} / \\
/ \mathrm{m} / / \mathrm{n} / / \mathrm{l} /\end{array}$ & $\begin{array}{l}/ \mathrm{b} / / \mathrm{j} / / \mathrm{p} / / \mathrm{tt} / / \mathrm{k} / \\
/ \mathrm{m} / / \mathrm{n} / / \mathrm{n} / / / /\end{array}$ & $\begin{array}{l}/ \mathrm{b} / / \mathrm{d} / / \mathrm{d} / / \mathrm{p} / / \mathrm{tt} / / \mathrm{k} / \\
/ \mathrm{m} / / \mathrm{n} / / \mathrm{n} / / \mathrm{r} / / / /\end{array}$ & $\begin{array}{c}/ \mathrm{b} / / \mathrm{d} / / \mathrm{d} / / \mathrm{g} / / \mathrm{p} / / \mathrm{tt} / / \mathrm{k} / \\
/ \mathrm{m} / / \mathrm{n} / / \mathrm{ln} / / \mathrm{r} / / / /\end{array}$ \\
\hline $90 \%$ & $/ \mathrm{b} / \mathrm{l} / \mathrm{ln} /$ & /j/ /I/ & $/ \mathrm{d} / / \mathrm{g} / / \mathrm{t} / / / \mathrm{r} / / \mathrm{II}$ & $/ \mathrm{d} / / \mathrm{g} / / \mathrm{tg} / / \mathrm{ff} / / \mathrm{s} /$ & $/ \mathrm{g} / / \mathrm{tf} / / \mathrm{ff} /$ & $\mid \mathrm{t} / / \mathrm{s} / / \mathrm{f} /$ \\
\hline $80 \%$ & $/ \mathrm{g} / / \mathrm{tg} / / \mathrm{k} / \mathrm{ff} / \mathrm{Ix} / \mathrm{ln} / \mathrm{Il} /$ & $/ \mathrm{d} / / \mathrm{g} / / \mathrm{t} / / / \mathrm{ff} / \mid \mathrm{x} / / \mathrm{r} /$ & $|f / / s /| x \mid$ & $|s| / x \mid$ & $|s||x|$ & $|x| / r \mid$ \\
\hline $70 \%$ & $\mid d / / c l$ & & $|r|$ & $|r|$ & - $|r|$ & \\
\hline $60 \%$ & $\mid \mathrm{s} /$ & & & & & \\
\hline $50 \%$ & & & & & & \\
\hline $40 \%$ & & & & & & \\
\hline $30 \%$ & & & & & & \\
\hline
\end{tabular}




\section{DISCUSION}

Considerando los resultados en su totalidad, las consonantes que presentaron mayor porcentaje de emisión normal fueron las nasales, sonoras continuas y sonoras no continuas. Esto coincide con los resultados entregados recientemente en una investigación en Río de Janeiro ${ }^{10}$ en la cual observaron que los fonemas /p/, /b/, /t/, /d/, / / /, /g/, /m/ y /n/ ya estarían adquiridos a los 3 años. En esta investigación todos estos fonemas (exceptuando $/ \mathrm{g} /$ con un $80 \%$ ) obtuvieron un $100 \%$ de articulación normal desde los 3 años de edad (Tabla 2).

Las dificultades articulatorias, en cambio estuvieron concentradas en las líquidas, específicamente vibrante múltiple y en las sordas continuas o fricativas. Lo anterior nos permitiría advertir una "secuencia" de adquisición fonológica en el niño, que concuerda con lo que habitualmente se ha señalado en la literatura clásica y algunos estudios sobre adquisición fonológica en el español ${ }^{6,7}$.

Resulta interesante observar que en una investigación realizada con sujetos que presentaban alteraciones en el desarrollo de su sistema fonológico ${ }^{11}$, los procesos fonológicos de simplificación de mayor ocurrencia - en cuanto a segmento- fueron precisamente aquellos que afectan a líquidas y fricativas, coincidente con los tipos de fonemas de más tardía adquisición en la presente investigación. La comparación anterior muestra que los segmentos de mayor complejidad articulatoria, y en consecuencia de más tardía adquisición están más expuestos a la acción simplificadora de los procesos fonológicos y por consiguiente a sustituciones fonológicas.

En algunas consonantes se observó un comportamiento irregular en el progreso hacia la articulación normal en relación al aumento de edad de los sujetos (ver líquidas en la Figura 6), no obstante, el rendimiento general de las consonantes presentó una tendencia al aumento de las emisiones normales en función del avance de edad en los informantes. El orden de adquisición de los segmentos encontrado en la presente investigación coincide con la línea evolutiva reportada para el español en una investigación anterior ${ }^{4}$. La secuencia observada mostró una adquisición más temprana para los segmentos nasales y oclusivos; y, más tardía para los fricativos y vibrante múltiple. Recientes investigaciones evidencian que la secuencia anterior tendría un carácter universal al ser observada en varias lenguas, por ejemplo en el inglés los primeros sonidos en aparecer son oclusivas, nasales y semiconsonantes. Cabe señalar, además, que esta "universalidad" se observa también en el tipo de estructura silábica predominante: $\mathrm{CV}^{4}$.
Otro aspecto interesante es la relación existente entre la disminución de las producciones no normales en función del avance de la edad, observada en esta investigación, y en el mejor rendimiento en pruebas de conciencia fonológica y de manejo de reglas fonológicas descritas por una investigación en niños peruanos recientemente ${ }^{12}$. Dichos investigadores muestran como las habilidades fonológicas se incrementan entre los 3 y 5 años de edad. En este mismo rango etáreo nuestra investigación evidenció un rápido desarrollo que a la edad de 5 años muestra una adquisición fonológica de sobre un $95 \%$.

La correlación entre edad y rendimiento fonológico también está presente en la disminución paulatina de los fenómenos articulatorios que el test CEFI considera como no normales: modificación y sustitución. La sustitución correspondió al fenómeno no normal con mayor porcentaje de aparición en la muestra y su presencia se mantiene aún en los informantes de mayor edad (V y VI grupos etáreos). El fenómeno de modificación tuvo una aparición homogénea en cuanto al porcentaje alcanzado en cada grupo donde se presentó. Cabe recordar que se considera articulación "modificada" a aquélla que constituye un cambio de tipo articulatorio, por esta misma razón, e independiente de las causas por las cuales se produjeron éstas emisiones, se debe aclarar que generalmente fueron emitidas por un mismo sujeto, quien "modificaba" un segmento en la posición en que éste se encontrase, ya sea de manera constante o inconstante. Este fenómeno indudablemente afectó los porcentajes totales del grupo etáreo del informante en cuestión. Situación similar ocurrió con la sustitución y omisión, aunque estos dos fenómenos presentaron un comportamiento más regular. Lo planteado anteriormente, obedece a que el desarrollo infantil en todos sus ámbitos presenta una gran variabilidad entre los sujetos, por lo cual es esperable encontrar casos que escapen al comportamiento general, ya sea de manera positiva o negativa. Cabe señalar que en este estudio, a pesar de estos casos aislados, se mantiene una tendencia en los resultados generales.

Con los datos obtenidos, no sería pertinente establecer una secuencia de adquisición cronológica estricta de los sonidos, pues los niños presentan variabilidad en el desarrollo fonológico y en la adquisición de las clases de sonidos. Son variados los elementos que pueden estar involucrados en este proceso de adquisición: "si bien a priori parece aceptable el hecho de plantear la noción de dificultad para la correcta realización de determinados sonidos, sobre todo si tenemos en cuenta el movimiento articulatorio involucrado o la precisión y velocidad requeridas en el habla fluida (...)" ${ }^{4}$. Esta 
misma autora suma al factor anterior, la influencia que puede tener la frecuencia de aparición que tienen los sonidos en una determinada lengua en la adquisición fonológica.

\section{CONCLUSIONES}

A partir de los 3 años de edad, el $80 \%$ de las articulaciones se producen normalmente. Sin embargo, los sujetos de 5,11 años aún no alcanzan a completar el sistema fonológico de manera normal. En otras palabras, los resultados indican que en pocos años (3) se produce una adquisición rápida de fonemas $(80 \%)$ para luego lentificarse en los siguientes tres años (20\%).

Las mayores dificultades de adquisición se manifestaron en las consonantes fricativas /s/ y / $/$ $y$, sobre todo, en la consonante $/ r /$.
A la inversa, las consonantes que menos dificultades de adquisición presentaron fueron las nasales y las oclusivas.

Por otra parte, el fenómeno de sustitución fue el que se presentó de manera más frecuente seguido de modificación y, finalmente, de omisión. En otras palabras, existe mayor confusión de fonemas que manifestación de variantes.

En general, se observa que los cambios mantienen un cierto equilibrio fonológico, es decir, una vibrante múltiple era sustituida por una lateral, por una aproximante $/ \mathrm{d} /$, por las dentales $/ \mathrm{d} / \mathrm{y} / \mathrm{t} /$, por la vibrante simple $/ \mathrm{r} /$ (todas de la zona dentoalveolar) y nunca por una labial o una velar. Si cambian la zona, por ejemplo, /f/ por /x/ ([fwé.yo] por [xwé.yo] mantienen el modo fricativo o si cambian $/ \mathrm{b} / \mathrm{por} / \mathrm{g} /$ [go.ro] por [bo.ro] mantienen el modo oclusivo.

\begin{abstract}
Purpose: to become familiar with the way children acquire the phonetic-phonological system in their maternal language and to describe the chronology of the acquisition sequence related to the phoneticphonological repertoire in the sample. Methods: the sample was composed of 72 individuals divided into six age groups of six months each. The "Questionnaire for Evaluating Child Phonology" (QECP) was applied in order to evaluate articulation of vowels in every position by means of a naming task. The results for each age group were compared in every segment in order to observe the acquisition sequence of sounds in every subject. Results: It was observed that the general performance of vowels showed an increase in the percentage of normal rendering in relation to the increase in age of the subjects. The phonemes $/ \mathrm{f} /-/ \mathrm{s} /-/ \mathrm{x} /$ and the group of liquids $/ / /-/ \mathrm{h} /-/ \mathrm{r} /$ were the segments that showed the highest articulatory difficulty. Nasals $/ \mathrm{m} /-/ \mathrm{n} /-/ \mathrm{nh} /$ and voiceless non continuous $/ \mathrm{p} /-/ \mathrm{t} / \mathrm{-} / \mathrm{tch} / \mathrm{-} / \mathrm{k} /$ were the segments that had the highest percentage of normal articulation. Conclusions: at the age of 3 , the subjects have over $80 \%$ of normal consonant articulation. The biggest acquisition difficulties were shown by fricative consonants $/ \mathrm{s} /$ and $/ \mathrm{x} /$, and, particularly, the consonant $/ \mathrm{r} /$. On the contrary, the consonants showing the lowest acquisition difficulty were the nasal and occlusive consonants.
\end{abstract}

KEYWORDS: Child Language; Language Development; Child Articulation; Phonetics

\section{REFERENCIAS}

1. Lleó C. La adquisición de la fonología de la primera lengua y de las lenguas extranjeras. Modelos teóricos y métodos linguísticos de análisis. Madrid: Visor; 1997. p. 15-40.

2. Stoel-Gammon C, Sosa AV. Phonological development. En: Hoff E, Shatz M. Blackwell handbook of language development. Blackwell Handbook; 2007.p. 238-256.
3. Locke JL. Gradual emergence of developmental language disorders. J Speech Hear Res. 1994; 37(3):608-16.

4. Bosch L. La evaluación fonológica del habla infantil. Barcelona: Masson; 2003. p. 35-42.

5. China N. La sonoridad y la marcación en los errores comunes del habla infantil. Rev Arg Neuropsicol. 2004; (4):23-37.

6. González MJ. Análisis del desarrollo fonológico en sujetos malagueños. Infancia y Aprendizaje. 1989; (48):3-24. 
7. Serra M. Normas estadísticas de articulación para la población escolar de 3 a 7 años del área metropolitana de Barcelona. Rev Logoped Fonitr Audiol. 1984; 3(4):232-5.

8. Pávez MM. Procesos fonológicos de simplificación en niños: su incidencia en los trastornos del lenguaje. Rev Linguist Toer Apl. 1990; (28):123-34.

9. Vivar $\mathrm{P}$, León $H$. Aplicación del cuestionario para la evaluación de la fonología infantil (CEFI) a una muestra de niños chilenos de diferente nivel socioeconómico. Rev Chilena Fonoaudiología. 2007; 8 (En Prensa)
10. Ferrante C, Van Borsel J, Medeiros M. Aquisição fonológica de crianças de clase sócio económica alta. Rev CEFAC. 2008; 10(4):452-60.

11. Kalil L, Takiuchi N. Prevalência das alterações fonológicas e uso dos processos fonológicos em escolares aos 7 anos. Rev CEFAC. 2008; 10(2):158-67.

12. Dioses A, Garcia L, Matalinares M, Cuzcano A, Panca N, Quiroz J, et al. Análisis psicolingüístico del desarrollo fonético-fonológico de alumnos preescoles de Lima Metropolitana. Rev Investigación en Psicología. 2006; 9(2):9-32.

RECEBIDO EM: 28/05/2008

ACEITO EM: 16/01/2009

Endereço para correspondência:

Pilar Vivar

Facultad de Humanidades y Arte

Departamento de Español

Universidad de Concepción

Rua Chacabuco sin numero

Concepcion - Chile

CEP: 4030000

E-mail: p_vivar_v@hotmail.com ; hleon@udec.cl 